\title{
EDUCAÇÃO EM SAÚDE NO AMBIENTE HOSPITALAR PEDIÁTRICO
}

\section{HEALTH EDUCATION IN THE PEDIATRIC HOSPITAL ENVIRONMENT}

\section{EDUCACIÓN EN SALUD EN EL MEDIO AMBIENTE HOSPITALARIO PEDIÁTRICO}

\author{
Renata Gonçalves ${ }^{1}$, Fernanda Carolina Camargo ${ }^{2}$, Maria Paula Custódio Silva ${ }^{3}$, Álvaro da \\ Silva Santos ${ }^{4}$, Jesislei Bonolo do Amaral ${ }^{5}$, Divanice Contim ${ }^{6}$
}

Como citar esse artigo: Gonçalves R, Camargo FC, Silva MPC, Santos AS, Amaral JB, Contim D. Educação em saúde no ambiente hospitalar pediátrico. Rev Enferm Atenção Saúde, v. 9, n. 2, 39-50, 2020. DOI: 10.18554/reas.v9i2.3558

\section{RESUMO}

Objetivo: Analisar significados atribuídos por enfermeiros sobre as práticas de educação em saúde no ambiente hospitalar pediátrico. Método: Empreendeu-se abordagem qualitativa em estudo exploratório descritivo, conduzido por entrevistas individuais junto a enfermeiras atuantes na unidade de internação pediátrica de hospital público ensino, setembro a novembro de 2017. Resultados: Conforme análise temática de conteúdo emergiram três categorias: Importância da educação em saúde na assistência à criança hospitalizada; Desenvolvimento da educação em saúde no ambiente hospitalar, Dificuldades e desafios no desenvolvimento da prática de educação em saúde na unidade hospitalar pediátrica. Os significados desvelaram fragmentações relacionadas as ações educativas para continuidade do cuidado em rede, resistências dos familiares ou responsáveis pelas crianças internadas e a necessidade de incremento na qualificação profissional. Conclusão: Apesar de a pesquisa limitar-se a única unidade de internação hospitalar pediátrica, os significados potencialmente podem ser generalizados a enfermeiros em contextos similares.

Descritores: Educação em Saúde; Enfermagem Pediátrica; Assistência Hospitalar; Cuidado da Criança.

\footnotetext{
ABSTRACT

Objective: To analyze meanings attributed by nurses on the practices of health education in the pediatric hospital environment. Method: Qualitative approach was undertaken in a descriptive exploratory study, conducted by individual interviews with nurses working in the pediatric hospitalization unit of a public teaching hospital, from September to November 2017. Results: According to the thematic content analysis, three categories emerged: Importance of health education in hospitalized child care; Development of health education in the hospital environment, Difficulties and challenges in the development of the practice of health education in the pediatric hospital unit. The meanings revealed fragments related to educational actions for continuity of care in the network, resistance of the relatives or responsible for hospitalized

${ }^{1}$ Enfermeira especialista em Saúde da Criança e do Adolescente. UFTM. Uberaba-MG. https://orcid.org/00000002-2673-9212

${ }^{2}$ Doutora em Enfermagem. UFTM. Uberaba-MG. https://orcid.org/0000-0002-1048-960X.

${ }^{3}$ Enfermeira especialista em Neonatologia e Mestranda em Atenção à Saúde. UFTM. Uberaba-MG. http://orcid.org/0000-0001-8694-1589

${ }^{4}$ Doutor em Enfermagem. UFTM. Uberaba-MG. https://orcid.org/0000-0002-8698-5650

${ }^{5}$ Doutora em Enfermagem. UFTM. Uberaba-MG. https://orcid.org/0000-0002-0591-7972

${ }^{6}$ Doutora em Enfermagem. UFTM. Uberaba-MG. https://orcid.org/0000-0001-5213-1465
} 
children and the need for an increase in professional qualification. Conclusion: Although the research is limited to the only unit of pediatric hospital admission, the meanings can potentially be generalized to nurses in similar contexts.

Descriptors: Health Education; Pediatric Nursing; Hospital Care; Child Care.

\section{RESUMEN}

Objetivo: Analizar significados atribuidos por enfermeros sobre las prácticas de educación en salud en el ambiente hospitalario pediátrico. Método: Se emprendió un abordaje cualitativo en un estudio exploratorio descriptivo, conducido por entrevistas individuales junto a enfermeras actuantes en la unidad de internación pediátrica de hospital público enseñanza, septiembre a noviembre de 2017. Resultados: Conforme análisis temático de contenido surgieron tres categorías: Importancia de la educación en salud en la asistencia al niño hospitalizada; Desarrollo de la educación en salud en el ambiente hospitalario, Dificultades y desafíos en el desarrollo de la práctica de educación en salud en la unidad hospitalaria pediátrica. Los significados desvelaron fragmentaciones relacionadas con las acciones educativas para la continuidad del cuidado en red, resistencias de los familiares o responsables por los niños internados y la necesidad de incremento en la calificación profesional. Conclusión: A pesar de que la investigación se limita a la única unidad de internación hospitalaria pediátrica, los significados potencialmente pueden ser generalizados a enfermeros en contextos similares.

Descriptores: Educación en Salud; Enfermería Pediátrica; Atención Hospitalaria; Cuidado del Niño.

\section{INTRODUÇÃO}

As atividades do enfermeiro têm se diversificado e ampliado, tornando-se um processo complexo, compreendido pelo cuidar, educar e gerenciar. Entretanto, na prática, tem-se observado uma visão limitada do cuidado por alguns enfermeiros que o desenvolve de forma fragmentada, voltado a uma assistência focada na resolução de problemas procedimentais. ${ }^{1}$

Acredita-se que a educação associada ao cuidado resulta na transformação do conhecimento, na construção e reformulação no âmbito das necessidades individuais e coletivas. ${ }^{2}$ Nesse sentido, a educação em saúde perpassa o cotidiano do enfermeiro, quanto a recuperação, prevenção e as necessidades de ensino do paciente. Essa realidade inclui o âmbito hospitalar, onde este profissional é chamado a reconstruir sua prática de cuidado direto, para um modelo mais abrangente, em que a educação faz parte da assistência. ${ }^{1-3}$

A educação em saúde destaca-se pela busca de uma relação dialógica entre profissionais e população, visando à participação cidadã e à formação de pessoas com autonomia e comprometidas com a coletividade. Como exemplo, o controle e prevenção de doenças, melhoria da qualidade de vida e tratamento de agravos, sendo considerada uma estratégia essencial no processo de conscientização individual e coletiva da sociedade. ${ }^{3,4}$

A educação em saúde ganha um papel importante para a enfermagem atingir seus 
objetivos no ambiente hospitalar. Este local deixa de ser uma instituição onde se restabelece a saúde de seus usuários para ter uma função mais abrangente na recuperação, manutenção e prevenção de doenças, e deve ser realizada pelo enfermeiro de maneira articulada aos objetivos educativos propostos para melhorias das condições de saúde. Isto ressalta que essas questões refletem o momento histórico da profissão, que busca, entre outras questões, compreender melhor os potenciais do ato educativo. .,2,5 $^{1}$

No cuidado à criança encontram-se espaços para o desenvolvimento de ações voltadas a educação em saúde. O processo educativo deve permear todas as práticas do cuidado infantil e envolver seus familiares de forma ativa diante das práticas dos profissionais de saúde, e desenvolver ações educativas que partam da realidade por ela vivida. $^{5-7}$

Nessa direção as interações educativas buscam a construção de uma relação verdadeiramente dialógica, tornando essa prática inerente e indissociável ao cuidado hospitalar, numa perspectiva de ação-reflexão-ação conscientizadora. Ao desenvolver as ações educativas por essa perspectiva, é induzido aos enfermeiros a revisão da sua identidade, enquanto educador, auxiliando na transformação, autonomia e emancipação dos indivíduos. ${ }^{6,7}$

Acredita-se ser esta práxis uma das formas de valorizar a profissão de enfermagem e ampliar o espaço de atuação profissional. ${ }^{1,5,8}$ Em se tratando de assistir a criança, a presença dos familiares, geralmente, promove e mantém a interrelação criança/família, neutraliza os efeitos decorrentes da separação de seus membros, colabora na assistência integral à criança, melhora sua adaptação ao hospital, facilita a aceitação do tratamento, promove positiva resposta terapêutica e ameniza os fatores estressantes da doença, dos procedimentos e da hospitalização. ${ }^{1,5,6}$ Desse modo este estudo tem como questão norteadora: Qual o significado atribuído pelos enfermeiros sobre as práticas de educação em saúde no ambiente hospitalar? Para responder ao questionamento, delineou-se o seguinte objetivo: analisar os significados atribuídos por enfermeiros sobre as práticas de educação em saúde no ambiente hospitalar pediátrico.

\section{MÉTODO}

Trata-se de um estudo descritivo exploratório de abordagem qualitativa. Esta escolha ocorreu uma vez que se baseia na abordagem interpretativa da realidade observada, com o intuito de se acessar o mundo subjetivo individual para a 
compreensão de significados que as pessoas constroem com base no que experienciam. ${ }^{9}$ Com isso, a abordagem qualitativa melhor atende aos objetivos da pesquisa e permite apreensão das opiniões e motivações expressas de enfermeiros em clínica pediátrica.

A pesquisa foi desenvolvida em um Hospital Universitário público referência para a alta complexidade hospitalar para a macrorregião Triângulo Sul do Estado de Minas Gerais. O estudo foi realizado na Unidade de Internação Pediátrica. Essa unidade tem como característica principal a internação de crianças e adolescentes para investigação diagnóstica e tratamento. Atuam nesta unidade equipe multiprofissional composta por médicos pediatras, fisioterapeuta, fonoaudiólogo, psicólogo, assistente social, enfermeiro e técnico de enfermagem. Nessa unidade são desenvolvidos, programas de residências: médica, enfermagem e multiprofissional e ainda mesma dispõe de recursos tecnológicos necessários para cuidados de média e alta complexidade.

As participantes do estudo foram enfermeiras que atuavam na unidade pediátrica em turnos de trabalho distintos. Como critérios de inclusão, elencaram-se enfermeiras assistenciais lotadas e atuantes há mais de um ano na assistência à criança hospitalizada. Quanto aos critérios de exclusão, as que estavam afastadas ou de férias no período da realização da coleta de dados.

As entrevistas foram previamente agendadas, conforme a disponibilidade dos participantes. Após a assinatura do Termo de Consentimento Livre e Esclarecido (TCLE), as enfermeiras foram entrevistadas individualmente e, em sala previamente reservada, com gravação em aparelho de áudio. O tempo para cada entrevista não foi delimitado. A definição do número de participantes ocorreu por inclusão progressiva, sendo estabelecido pelo critério de saturação, isto é, quando a opinião das participantes sobre o assunto começou a expressar regularidade de apresentação, além de responder ao objetivo do estudo.

A coleta dos dados foi realizada entre os meses de outubro a dezembro de 2017, a partir do desenvolvimento da técnica de entrevista semiestruturada. A primeira parte foi composta pelo levantamento dos dados sócio demográficos: gênero, idade, tempo de formação, tempo de experiência, tempo de serviço nesta instituição, formação profissional, carga horária semanal nesta instituição, turno de trabalho. Na segunda parte foram questionados os significados das práticas de educação em saúde.

Para o tratamento das narrativas resultantes das entrevistas, adotou-se a 
análise de conteúdo temática ${ }^{9}$, que consiste em um agrupamento de técnicas, passando pelas fases de pré-análise, exploração do material e tratamento dos resultados, inferência e interpretação. Foram realizadas leituras consecutivas, com vistas a sistematizar os dados. Na primeira leitura, efetuou-se o contato exaustivo com o material sinalizando os pontos de interesse. Os dados importantes foram destacados para garantir que não fossem descartados.

$\mathrm{Na}$ leitura seguinte, realizou-se a codificação dos dados, organizados segundo unidades de significado, com a finalidade de visualizá-los de forma agrupada de acordo com os objetivos da pesquisa. $\mathrm{Na}$ fase de exploração, os dados foram categorizados.

Para garantir o sigilo e confidencialidade das enfermeiras, optou-se pela utilização da letra 'E', por ser a letra inicial da área em que este estudo se inscreve, Enfermagem, seguidas de um número, indicando o número de participantes da pesquisa, E1, E2..., E5.

O estudo atendeu as exigências éticas presentes na Resolução $n^{\circ} 466 / 2012$ do Conselho Nacional de Saúde, o projeto foi aprovado pelo Comitê de Ética em Pesquisa (CEP) com seres humanos da Universidade Federal do Triângulo Mineiro sob CAAE: 65821617.7.0000.5154.

\section{RESULTADOS}

Participaram da pesquisa cinco enfermeiras com idade entre 33 e 61 anos. O tempo de formação foi entre 12 e 25 anos e o tempo de serviço na instituição variou entre dois e 25 anos. Das participantes uma possui mestrado, três possuem especialização e uma não informou. A carga semanal de trabalho das participantes era de 36 horas. Uma das enfermeiras trabalha no período matutino, três no período vespertino e, uma no período noturno.

A partir da análise e interpretação das entrevistas emergiram três categorias temáticas, denominadas: importância da educação em saúde na assistência à criança hospitalizada; desenvolvimento da educação em saúde no ambiente hospitalar; e, dificuldades e desafios no desenvolvimento da prática de educação em saúde na unidade hospitalar pediátrica.

\section{Importância da educação em saúde na assistência à criança hospitalizada \\ Essa categoria representa a} importância da educação em saúde para enfermeiras frente essa prática no ambiente hospitalar. Nos relatos registram-se a relevância dessa estratégia como recurso para a melhoria do serviço prestado pela equipe e a continuidade do cuidado pelo responsável da criança após a alta.

Super importante, [...] Em todas as unidades eu acho, porque cada dia está tendo doenças umas 
diferentes das outras e os cuidados são diferentes $[\ldots](E 2)$

É importante para estar deixando o pessoal sempre preparado [...] É importante também para orientar as mães na alta da criança, no cuidado que ela vai ter no domicílio [...] (E5)

Orientar as mães no cuidado também em relação às doenças [...] Doenças com as crianças [...] Em relação aos profissionais para eles realizarem um melhor trabalho, [...] de forma mais efetiva. (E4)

\section{Desenvolvimento da educação em saúde no ambiente hospitalar}

Esta unidade temática refere-se à forma como os enfermeiros da unidade pediátrica se percebem desenvolvendo a educação em saúde no seu cotidiano de trabalho.

Eu vejo a educação em saúde de duas formas [...] aquela que você orienta e ensina mesmo os acompanhantes [...] a família que fica com a criança o tempo todo [...] na parte de orientações, de como você vai conduzir a família o acompanhante para o ambiente hospitalar [...] fazer que esse familiar, esse acompanhante veja as orientações e ensinamentos oferecidos durante a internação seja para o cuidado fora do hospital [...] que se habitue a cuidar da criança de forma adequada. (E5)

O desenvolvimento da educação em saúde por meio da educação permanente e ou em serviço foi uma das formas evidenciadas nas falas das enfermeiras.

Então aqui a gente faz um treinamento com a equipe de enfermagem todo mês [...] (E1)

Eu penso que educação em saúde é você orientar, ensinar e atualizar os profissionais em relação às doenças, as rotinas, procedimentos do setor. (E4)
O desenvolvimento da educação em saúde seria mais efetivo se fosse diretivo realizado na unidade, isso em relação aos profissionais [...] Isso poderia ser feito com a participação de toda a equipe. (E4)

Quanto às orientações oferecidas aos familiares e acompanhantes, principalmente na alta hospitalar, também foram referidas como prática de educação em saúde realizada pelas enfermeiras.

Você vai orientar a mãe a cuidar da criança [...] falar sobre a doença o que pode ser feito [...] você pode desenvolver uma estratégia individualizada para aquela criança [...] aquela necessidade [...] para evitar [...] para evitar complicações. (E1)

O desenvolvimento da educação em saúde poderia ser focada na alta. Para mim essa educação começa quando a criança é admitida no hospital [...] você vai ensinado, orientando, supervisionando $e$ assim você planeja a alta [...]

Quando sair de alta e for para casa dependendo do que você for orientar a mães vai estar mais preparada com relação ao cuidado com a criança. (E4)

\section{Dificuldades e desafios no desenvolvimento da prática de educação em saúde na unidade hospitalar pediátrica}

Dentre os desafios referidos pelas enfermeiras participantes está a falta de tempo, de material e local adequado para o desenvolvimento da educação em saúde.

Então falta de tempo mesmo (...) é muita coisa para gente fazer, não dá tempo (...) (E1)

Em relação à parte técnica a gente não tem um local adequado para fazer essa educação, não tem material adequado, equipamentos. (E2) 
Eu percebo alguns desafios sobre o desenvolvimento da educação em saúde na unidade pediátrica [...] Acho que tem uma dificuldade quanto ao interesse dos familiares sobre as informações que são passadas [...] eu não sei te dizer se é falta de preparo por parte dos profissionais, se é um processo de comunicação ineficiente por parte da equipe [...] porque às vezes não valoriza a informação [...] a orientação que é oferecida. (E2)

A dificuldade de entendimento por parte de familiares ou responsáveis, pela criança, também foi citada como uma barreira na realização das ações de educação em saúde.

Porque eles têm muita dificuldade de assimilar até orientações básicas. (E2)

A dificuldade muita vezes que eu encontro e na hora de tá falando com essa família, eles às vezes tem um conhecimento muito restrito, né? (E3)

Parece que a pessoas não aprende, não absorve nem vai colocar em pratica o que você falou. (E4)

A fala das participantes demonstram uma visão de educação em saúde como uma ação que deveria ser mais elaborada. A ser trabalhada por grupos especializados criados para isto.

Em relação aos pais é mais uma tirada de dúvidas mesmo, a gente não faz uma educação em saúde com eles não. ( E2)

A ideia é ter um grupo de educação com a mãe [...] Até a questão da humanização também. (E2)

É [...] fazendo até uma simulação com um boneco, [...] a gente não tem aqui essa parte de fazer a simulação. (E5)

\section{DISCUSSÃO}

Nas entrevistas, nota-se que a percepção da educação em saúde é importante para a melhoria dos serviços e da continuidade do cuidado no hospital e no domicílio. As participantes apontaram que no cotidiano do trabalho do enfermeiro a utilização da educação em saúde como ferramenta assistencial, porém fragmentada e realizada de forma pontual, desenvolvida separadamente do cuidado diário. Desse modo, apesar do papel de educador ser inerente ao trabalho enfermeiro $\mathrm{e}$ a educação em saúde ser ferramenta importante para prática assistencial, observa-se que seu desenvolvimento ocorre de forma reducionista, com orientações individualizadas, prescritivas e focadas na doença. $^{10-12}$

A participantes identificaram a educação em saúde como importante ferramenta para a melhoria dos serviços e da continuidade do cuidado em domicílio, no entanto não abordam a necessidade de articulação junto a Atenção Primária á Saúde (APS). Contudo, estudos indicam enfermeiro deve apresentar conhecimento e habilidade para auxiliar os usuários em momentos de agravos, bem como orienta-lo e capacita-lo para identificar precocemente alterações que possam surgir no domicílio. ${ }^{12,13,14}$ Vale ressaltar que estudos indicam que essa articulação pode ser viabilizada por meio do sistema de 
referência e contra referência durante o planejamento da alta hospitalar. ${ }^{14,15}$

Nesse estudo as participantes compreendem que o momento de internação é ímpar na possibilidade de orientações por meio de ações da educação em saúde. Entendem que os pais e responsáveis pelas crianças valorizam o aprendizado do cuidado durante a hospitalização. Porém destacam a falta de recursos didáticos como um ponto negativo, tornando o aproveitamento pouco eficaz durante a realização dos cuidados diretos com criança, dificultando a compreensão dos familiares e responsáveis na aprendizagem desses ensinamentos. ${ }^{8,15}$ Desse modo as orientações fornecidas por meio processos educativos perpassa por atuações pontuais como a necessidade de fornecer orientações sobre o estado de saúde dos filhos com a finalidade de garantir a continuidade do cuidado após alta. No entanto as participantes destacaram que falta de entendimento dos familiares quanto às orientações passadas durante a hospitalização e na preparação da alta das crianças, podem estar relacionada a pouca valorização que as próprias enfermeiras atribuem a tal atividade ${ }^{8,10,15,16}$

Dentre os cuidados básicos associados a educação em saúde no ambiente hospitalar as participantes destacam-se a puericultura, a imunização, os cuidados básicos do cotidiano da criança. Desse modo percebe-se, a importância de ações práticas para a promoção da integração com a rede básica de atenção a saúde. ${ }^{17,18,19}$

Sobre a formação as enfermeiras entrevistadas revelaram que durante a graduação foram oferecidos conteúdos sobre educação em saúde direcionado para o cuidado integral como uma atribuição da atenção primaria. No entanto estudos indicam que a educação não é atributo exclusivo da atenção primaria e que as ações de educação em saúde podem e devem ser realizadas e que o hospital, pois este ambiente possibilita a construção, a desconstrução e a reconstrução dos conhecimentos individuais e coletivos. ${ }^{3,5,19}$

Nos depoimentos foi possível evidenciar a sobrecarga de trabalho como um fator que interfere de forma negativa das práticas de educação em saúde em seu local de trabalho. Este fato está vinculado a constituição e organização histórica do ambiente hospitalar, mantidas por relações de poder rígidas, voltada para o tecnicismo e medicalização da saúde, que foram sendo naturalizados no espaço hospitalar pelo discurso biomédico. ${ }^{3,12-13}$

Nessa direção, partindo da premissa que para uma informação ser efetiva deve ser baseada no compartilhamento de saberes que favoreça uma relação de 
vínculo e confiança entre cuidador e o profissional para que haja a mudança; e que pode ser prejudicado quando o profissional se coloca na condição do detentor do saber e adota uma postura autoritária, podendo resultar na inibição do usuário tornando-o passivo e não participativo no processo de cuidar. ${ }^{13}$ Situações onde o usuário se encontra em condições prescritivas e/ou impositivas desestimulam o seu aprendizado. $5,10,12$

Dificuldades concretas como a falta de tempo, de material, ambiente adequado na realização das atividades educativas também foram citadas na pesquisa. Este fato é respaldado por outros estudos onde as ações de educação de saúde são desenvolvidas no modelo tradicional de ensino, caracterizado por ações pontuais, fragmentadas e que enfrentam dificuldades de infraestrutura, material, gestão, recursos e sobrecarga de trabalho. ${ }^{17-19}$

Nos depoimentos das enfermeiras sobre o desenvolvimento das ações de educação em saúde, observa-se que a orientação e os ensinamentos aos acompanhantes são decisivos para superação dos cuidados domiciliares, na expectativa de contribuir para a melhoria da qualidade do cuidado contínuo, favorecendo as atividades do cotidiano e a relação entre profissional e usuário. Refletindo em um cuidado integral, humanizado que abrange aspectos físico, mental, ambiental, pessoal e social. ${ }^{15,17-20}$

Vale registrar que nas entrevistas as enfermeiras referiram que $o$ desenvolvimento da educação em saúde deve ser voltado a capacitações dos membros da equipe. Tem-se aqui um equívoco que é conceitual, semântico e, portanto, de prática. Ações educativas com profissionais de saúde estão na esfera da educação permanente. Ou seja, na prática quando se fala de educação em saúde se fala de ações educativas com usuários, clientes, pacientes e não com profissionais de saúde. ${ }^{4,10,12}$

\section{CONCLUSÃO}

Os significados atribuídos pelas enfermeiras participantes dessa pesquisa têm como foco a educação em saúde voltada para a família da criança hospitalizada e educação permanente e, o quanto essa se relaciona a prática assistencial de enfermagem, sinalizando a importância do apoio institucional e a da interação entre a equipe de saúde.

Verificou-se num discurso de valorização das ações de educação em saúde, porém observa-se descrédito na aceitação do familiar da criança quanto às orientações sobre o agrava e alta. Registram-se erros conceituais, desconhecimento sobre a prática de ações 
de contra referência, aspectos estes que podem ser reflexos da formação e do próprio ambiente hospitalar. Há necessidade de se rever os conceitos sobre a abrangência da educação em saúde no intuito de que o enfermeiro amplie suas competências para assumir este fazer como uma ação de cuidado contínuo, para além de o espaço hospitalar.

O estudo apresenta como limitação o fato de ter sido realizado em uma unidade pediátrica de um hospital geral de ensino, potencialmente o método empreendido permite generalizações à enfermeiros em contextos similares. Os resultados apoiam a identificação dessa realidade, quanto as fragmentações relacionadas ao desenvolvimento dos atos educativos, vivenciados neste cotidiano institucional. Quanto ao método é importante frisar, quanto ao número de participantes, que apesar de serem apenas cinco enfermeiras, elas corresponderam a quase totalidade de enfermeiros atuantes no setor. Contudo, os resultados apresentados, a aproximação de significados apoia o desvelamento de lacunas e potencialidades para o incremento da prática educativa no ambiente hospitalar pediátrico.

\section{REFERÊNCIAS}

1. Figueira AB, Amestoy SC, Cecagno D, Tristão FS, Trindade LL, Correa VÁ. Visão do enfermeiro frente à prática da educação em saúde no ambiente hospitalar. Cogitare Enferm. [Internet]. 2013 [citado em 20 jul 2020]; 18(2):310-6. Disponível em:

https://revistas.ufpr.br/cogitare/article/view /32580/20695. doi:

http://dx.doi.org/10.5380/ce.v18i2.32580

2. Santili PGJ, Tonhom SFR, Marin MJS.

Health education: challenges for implementation. Atas CIAIQ [Internet]. 2016 [citado em 01 jun 2020]; 2:1147-56. Disponível em:

https://proceedings.ciaiq.org/index.php/ciai q 2016/article/viewFile/867/851

3. Nogueira IS, Vergaças HM, Santos LF, Cypriano PE, Moreno MG, Lima SO et al. A prática educativa na estratégia saúde da família: estratégia para repensar e reconstruir ações dialógicas. Arq Ciênc Saúde UNIPAR. [Internet]. 2015 [citado em 24 jul. 2020]; 19(1):11-17. Disponível em:

https://www.revistas.unipar.br/index.php/sa ude/article/viewFile/5259/3032

4. Laguna MRC, Abreu MTL. Educação em saúde: abordagens conceituais. In: Santos AS, Paschoal VDA, organizadores. Educação em saúde e enfermagem. Barueri, SP: Manole; 2017. p. 42-44. (Série Enfermagem).

5. Massara CL, Murta FLG, Enk MJ, Araújo AD, Modena CM, Carvalho OS.

Caracterização de materiais educativos impressos sobre esquistossomose, utilizados para educação em saúde em áreas endêmicas no Brasil. Epidemiol Serv Saúde [Internet]. 2016 [citado em 20 jul 2020]; 25(3):575-84. Disponível em: https://doi.org/10.5123/s167949742016000300013

6. Silva KS, Aguiar LC, Costa ACLF, Nascimento RKG. Educação em Saúde: reflexões a partir da vivência de residentes multiprofissionais. Tempus (Brasília) [Internet]. 2016 [citado em 20 jul 2020]; 10(4):283-8. Disponível em: www.tempus.unb.br/index.php/tempus/arti cl e/download/2268/1735

7. Monteiro MM, Silva VB; Alcântara A, Costa E. Educação e cuidado em saúde por 
meio do Arco de Maguerez na pediatria clínica. Atas CIAQ [Internet]. 2016 [citado em 20 jul 2020]; 2:49-56. Disponível em: https://proceedings.ciaiq.org/index.php/ciai q2016/article/view/736

8. Santos RA, Lopes VC, Camillo SO, Maiorino FT. Perceções do graduando de enfermagem sobre a importância do acompanhante do paciente internado. Rev enferm Cent-Oeste Min. [Internet]. 2015 [citado em 20 jul 2020]; 5(1): 1425-38. Disponível em:

http://www.seer.ufsj.edu.br/index.php/reco $\mathrm{m} /$ article/view/690/833. doi: https://doi.org/10.19175/recom.v0i0.690

9. Minayo MCS. O desafio do conhecimento: pesquisa qualitativa em saúde. 10ed. São Paulo: HUCITEC; 2014

10. Flores GEF, Oliveira DLL, Zocche DAA. Educação permanente no contexto hospitalar: a experiência que ressignifica o cuidado em enfermagem. Trab Educ Saúde [Internet]. 2016 [citado em 20 jul 2020]; 14(2):487-504. Disponível em: https://www.scielo.br/pdf/tes/v14n2/16781007-tes-1981-7746-sip00118.pdf. doi: http://dx.doi.org/10.1590/1981-7746sip00118

11. Lima VX, Brito MEM. Percepções da equipe de enfermagem acerca da prática da educação em saúde em um centro de tratamento de queimados. Rev Bras Queimaduras [Internet]. 2016 [citado em 20 jul 2020]; 15(2):110-15. Disponível em: http://www.rbqueimaduras.com.br/summar $\mathrm{y} / 34$

12. Pio FSCG, Azevedo DM, Marques LF, Santiago LC. Nursing care in heart transplantation: integrative review. Rev Enferm (Lisboa) [Internet]. 2016[citado em 20 jul 2020]; 5(10):1857-65. Disponível em:

https://periodicos.ufpe.br/revistas/revistaen fermagem/article/view/13566/16355. doi: https://doi.org/10.5205/1981-8963v10i5a13566p1857-1865-2016

13. Pereira MM, Rodrigues PF, Santos NCCB, Vaz EMC, Collet N, Reichert APS.

Educação em saúde para famílias de crianças/adolescentes com doença crônica.
Rev Enferm UERJ. [Internet]. 2017 [citado em 20 jul 2020]; 25:e4343. Disponível em: https://www.e-

publicacoes.uerj.br/index.php/enfermagem uerj/article/view/4343/25920. doi:

http://dx.doi.org/10.12957/reuerj.2017.434 3

14. Wachholz LF, Knihs NS, Martins SR, Magalhães ALP, Brehmer LCF, Martins

MS. Alta hospitalar do paciente transplantado hepático: revisão integrativa. Esc Anna Nery Rev Enferm. [Internet]. 2020 [citado em 20 jul 2020]; 24(4):e20190346. Disponível em: https://www.scielo.br/pdf/ean/v24n4/141 4-8145-ean-24-4-e20190346.pdf. doi: https://doi.org/10.1590/2177-9465-ean2019-0346

15. Chagas MCS, Gomes GC, Pereira FW, Diel PKV, Farias DHR. Meaning given by family to care for the hospitalized child. Av Enferm. [Internet] 2017 [citado em 20 jul 2020]; 35(1):7-18. Disponível em:

http://www.scielo.org.co/pdf/aven/v35n1/ v35n1a02.pdf. doi: 10.15446/av.enferm.v35n1.42466

16. Azevêdo AVS, Lançoni Júnior AC, Crepaldi, MA. Nursing team, family and hospitalized child interaction: an integrative review. Ciênc Saúde Colet. [Internet] 2017 [citado em 01 jun 2020]; 22(11):3653-66. Disponível em: https://www.scielo.br/pdf/csc/v22n11/en 1413-8123-csc-22-11-3653.pdf. doi: http://dx.doi.org/10.1590/1413812320172211.26362015

17. Gropp M, Johnson E, Bornman J, Koul R. Nurses' perspectives about communication with patients in an intensive care setting using a communication board: a pilot study. Health SA. [Internet] 2019 [citado em 01 jun 2020]; 24:1162. Disponível em: https://www.ncbi.nlm.nih.gov/pmc/article s/PMC6917450/. doi: http://dx.doi.org/10.4102/hsag.v24i0.116 2

18. Zanfolim LC, Ganassin FMH, Cerchiari EAN. Dificuldades Vivenciadas pelas 
Mães na Hospitalização de seus Bebês em Unidades Neonatais. Psicol Ciênc Prof. [Internet] 2018 [citado em 01 jun 2020]; 38(1):22-35. Disponível em:

https://www.scielo.br/pdf/pcp/v38n1/141 4-9893-pcp-38-01-0022.pdf. doi: https://doi.org/10.1590/19823703000292017

19. Reichert APS, Collet N, Eickmann SHE, Lima MC. Vigilância do desenvolvimento infantil: estudo de intervenção com enfermeiros da Estratégia Saúde da Família. Rev Latinoam Enferm. [Internet] 2015 [citado em 20 jul 2020]; 23(5):954-62.

Disponível em:

https://www.scielo.br/pdf/rlae/v23n5/pt_0 104-1169-rlae-23-05-00954.pdf. doi: 10.1590/0104-1169.0272.2636

20. Neves GBC, Andreto LM, Oliveira CR, Figueira MCS. Opinião dos enfermeiros sobre educação permanente em um hospital público. Rev Enferm UFPE on line [Internet]. 2016 [citado em 20 jul 2020]; 10(5):1625-34. Disponível em: https://periodicos.ufpe.br/revistas/revistaen fermagem/article/view/11158/12677

RECEBIDO: 05/02/2019

APROVADO: $26 / 08 / 2020$ PUBLICADO: $12 / 2020$ 